\section{0-20 USING PROJECT ECHOTM TO DELIVER PALLIATIVE CARE EDUCATION AND SUPPORT ACROSS MULTIPLE SETTINGS}

${ }^{1}$ Janet Diffin, ${ }^{1}$ Tracey McTernaghan, ${ }^{2}$ Aine McMullan, ${ }^{2}$ Martin Hayes, ${ }^{1}$ Max Watson. ${ }^{1}$ Hospice UK, London, UK; ${ }^{2}$ Health and Social Care Board, Belfast, UK

\subsection{6/bmjspcare-2019-HUKNC.20}

Background There is a need for high quality palliative care education and support, but often limited resources are available. Project $\mathrm{ECHO}^{\mathrm{TM}}$ (Extension for Community Healthcare Outcomes) is a tele-mentoring programme which could overcome this gap by using video-conferencing technology to deliver:

- Evidence-based, best practice guidance and education from specialists;

- Case-based learning with opportunities for questions and discussion with peers.

Five $\mathrm{ECHO}^{\mathrm{TM}}$ networks in Northern Ireland (NI) delivered palliative care education and support: Cardiology/heart failure; District nurses; Palliative care pharmacy; Paediatric palliative care; Marie Curie registered nurses. Network participants identified educational topics for their curriculum, and set network aims and objectives. Objectives included increasing participants' knowledge, skills and confidence in palliative care related areas, improving collaboration, and facilitating peer support. Network leads were responsible for evaluating how well objectives were met.

Aim To evaluate how successful each $\mathrm{ECHO}^{\mathrm{TM}}$ network was in achieving it aims and objectives.

Methods Retrospective online survey designed specifically for each network issued to registered participants after the final $\mathrm{ECHO}^{\mathrm{TM}}$ session.

Results Across the five networks, $45 \mathrm{ECHO}^{\mathrm{mm}}$ sessions were delivered with 194 participants attending at least two. 27\% (60/224) registered participants across each network responded to the survey. Overall, each network was successful in achieving its aims. The majority of participants reported increased knowledge and confidence in relation to the areas assessed. $\mathrm{ECHO}^{\mathrm{TM}}$ was identified as a suitable model for delivering education and the opportunity for case-based learning was valued. Direct impacts on practice included improved inter-agency working and networking, improved communication with patients and families, and improved confidence to manage similar cases in the future. Barriers to participation included time restraints due to staffing issues or workload.

Conclusion Project $\mathrm{ECHO}^{\mathrm{m}}$ may be an ideal methodology for delivering palliative care education and support to a range of practitioners. However, protected time for participation is recommended.

Funders Health and Social Care Board NI/Hospice UK.

\section{0-21 RECOMMENDATIONS FOR COMPREHENSIVE, PERSON- CENTRED CARER SUPPORT: UK SURVEY OF CURRENT PROVISION}

${ }^{1}$ James Higgerson, ${ }^{2}$ Gail Ewing, ${ }^{1}$ Christine Rowland, ${ }^{1}$ Gunn Grande. 'University of Manchester, Manchester, UK; ${ }^{2}$ University of Cambridge, Cambridge, UK

10.1136/bmjspcare-2019-HUKNC.21

Background Carers play a vital role in supporting patients at end-of-life (EOL) enabling care at home and preventing hospital (re)admission. EOL care policy promotes comprehensive person-centred assessment and support for carers, but without a clear implementation strategy this remains an aspiration.

Aims (1) To develop recommendations for organisational structures and processes required for implementation of comprehensive person-centred assessment and support for carers in EOL practice. (2) To identify the structures and processes currently in place to achieve assessment and support for carers of patients receiving EOL care through UK hospice organisations. Methods (1) Ten recommendations were constructed from the findings of secondary analysis of existing research data and extensive expert and stakeholder consultation. (2) All hospices providing adult services within Hospice UK's membership $(n=200)$ were eligible to participate in a cross-sectional survey between March and June 2018.

Results An organisational survey was developed to assess hospice provision against the 10 national recommendations published by Hospice UK (2018) for organisational change needed to deliver current EOL care policy guidance for comprehensive, person-centred assessment and support for family carers (Ewing \& Grande, 2018). $115 \quad$ (58\%) hospices responded to the survey. $37 \%$ used a formal carer assessment process; $13 \%$ reported a specific action plan for carers. Other recommendations met at a higher rate, included recording demographic carer data (95\%) and consistent identification of carers within hospices (87\%). Less frequently met were monitoring and auditing of carer support (31\%), and consistent storage of carer-specific data (18\%). Results for each recommendation will be presented.

Discussion Most organisations met recommendations for structures and processes to achieve assessment and support for carers, although comprehensiveness of provision varied, and formal assessment provision occurred at a low rate.

Conclusion Some recommendations are well established in UK hospices, though particular areas for improvement include the use of person-centred processes specific to carers: formal assessment, action plans, recording processes and monitoring systems.

\section{Parallel Session 6: Community Engagement and Collaboration}

\section{0-22 HOW CAN COMMUNITIES SUPPORT CARERS - DEVELOPING COMMUNITY CAPACITY AND CAPABILITY?}

Gina King, Claire Henry. Hospice UK, London, UK

10.1136/bmjspcare-2019-HUKNC.22

In Ambitions for Palliative and End of Life Care (2015) Ambition 6 states that the aspiration is that 'Each community is prepared to help'. The range of terms used to describe this approach are based on the principle that care at the end of life should be done with people and not to people, that death, dying, loss and care are complex social events, in which the medical component is only one aspect.

This paper outlines the outputs and future considerations from a series of workshops that took an Appreciative Inquiry Model (Definition, Discovery, Dream, Design, Delivery onto 